communication. Med Care Res Rev. 2013;70(Suppl):37S-49S. http://dx.doi.org/10.1177/1077558712458541

4. Peters G-JY, Ruiter RAC, Kok G. Threatening communication: a critical re-analysis and a revised meta-analytic test of fear appeal theory. Health Psychol Rev. 2013;7(Suppl 1):S8-31. http://dx.doi.org/10.1080/17437199.2012.703527

Address for correspondence: Brian J. Zikmund-Fisher, Department of Health Behavior and Health Education, University of Michigan, 1415 Washington Heights, Ann Arbor, MI 48109-2029, USA; email: bzikmund@umich.edu

\section{West Nile Virus Seroprevalence, Connecticut, USA, 2000-2014}

\author{
Megan E. Cahill, Yi Yao, David Nock, \\ Philip M. Armstrong, Theodore G. Andreadis, \\ Maria A. Diuk-Wasser, Ruth R. Montgomery
}

Author Affiliations: Yale University School of Public Health, New Haven, Connecticut, USA (M.E. Cahill); Yale University School of Medicine, New Haven (Y. Yao, D. Nock, R.R. Montgomery); The Connecticut Agricultural Experiment Station, New Haven (P.M. Armstrong, T.G. Andreadis); Columbia University, New York, New York, USA (M.A. Diuk-Wasser)

DOI: http://dx.doi.org/10.3201/eid2304.161669

West Nile virus (WNV) infection is mainly asymptomatic but can be severe in elderly persons. As part of studies on immunity and aging in Connecticut, USA, we detected WNV seroconversion in $8.5 \%$ of nonimmunosuppressed and $16.8 \%$ of immunosuppressed persons. Age was not a significant seroconversion factor. Our findings suggest that immune factors affect seroconversion.

Since the 1999 emergence of West Nile virus (WNV) in North America, $>43,000$ cases of disease and 1,884 deaths have been reported $(1)$; overall infections are estimated at $\approx 3$ million (2). Although WNV infections can be asymptomatic, they can also cause severe neuroinvasive disease, especially among infants, immunocompromised persons, and elderly persons (3). Control of WNV infection involves innate immune pathways that mediate initial recognition and regulation of viral replication and adaptive immune responses that provide long-term protection (3). Spatial distribution analysis and mosquito surveillance studies have confirmed that WNV is endemic to Connecticut, USA $(1,4)$.
We compared seroprevalence and demographics for 890 nonimmunosuppressed and 173 immunosuppressed adults enrolled in a study on immunity in aging (approved by the Human Investigations Committee of Yale University) (5) with those of symptomatic WNV case-patients reported to the Connecticut Department of Health (DPH) during 2000-2014. DPH-reported symptomatic case-patients $(\mathrm{n}=116)$ sought medical attention and had a positive WNV laboratory test result (1). None of the asymptomatic participants were reported to DPH as WNV case-patients. Immunosuppressed participants followed an immunosuppressive medication regimen or had a diagnosis of rheumatoid arthritis (5). For all participants, we assessed previous exposure to WNV by immunoblot for WNV envelope protein (6). Seroconversion to WNV was distinguished from cross-reactivity to other flaviviruses by rescreening all positive serum against a recombinant WNV-specific mutant envelope protein that lacks the conserved cross-reactive fusion loop epitope (7).

We compared demographic characteristics of participant groups by using the Student $t$-test for continuous variables and $\chi^{2}$ and Fisher exact tests for categorical variables; $\mathrm{p}<0.05$ indicated statistical significance. Analysis was completed with SAS software version 9.3 (SAS Institute, Cary, NC, USA) and Prism 6 (GraphPad Software, Inc., La Jolla, CA, USA).

Immunoblot detected evidence of WNV exposure in $76(8.5 \%)$ of the 890 nonimmunosuppressed participants (Table). These seropositive participants reported neither symptoms nor diagnosis of WNV infection and are considered to have had asymptomatic infections. Timing of asymptomatic infections could not be determined, but antibodies against $\mathrm{WNV}$ are durable and do not differ between asymptomatic and symptomatic adults (8).

Although age is a critical risk factor for severe WNV infection $(3,9)$, the mean age of seropositive and seronegative nonimmunosuppressed participants did not differ significantly (Table). The rate of asymptomatic seroconversion did not vary significantly among the 890 persons in 3 age groups: $<35$ years $(42 / 421), 35-65$ years $(7 / 121)$, and $>65$ years $(27 / 348)(p=0.338)$. Seroconversion rates did not differ significantly by patient sex but were significantly elevated among those in self-identified Hispanic groups $(p<0.0001)$, possibly because of different exposure histories. The similar age distribution among asymptomatic seroconverters suggests that the observed age-associated susceptibility to clinically apparent disease may result from other factors, including individual host factors and dysregulation in immune responses $(6,10)$.

Among 173 immunosuppressed adults, 29 (16.8\%) showed evidence of exposure to WNV (Table), resulting in 2.16 times the odds of positive immunoblot result than for nonimmunosuppressed adults $(76 / 890,8.5 \% ; \mathrm{p}=0.002)$. 
Table. Seroconversion rates among participants in study of West Nile virus, Connecticut, USA, 2000-2014*

\begin{tabular}{|c|c|c|c|c|c|}
\hline \multirow[b]{3}{*}{ Participant } & \multicolumn{4}{|c|}{ Asymptomatic } & \multirow{3}{*}{$\begin{array}{c}\text { Symptomatic, } \\
\text { DPH case-patients } \\
2000-2014, \mathrm{n}=116\end{array}$} \\
\hline & \multicolumn{2}{|c|}{ Not immunosuppressed } & \multicolumn{2}{|c|}{ Immunosuppressed } & \\
\hline & $\begin{array}{c}\text { Seropositive, } \\
n=76\end{array}$ & $\begin{array}{c}\text { Seronegative, } \\
n=814\end{array}$ & $\begin{array}{c}\text { Seropositive, } \\
n=29\end{array}$ & $\begin{array}{c}\text { Seronegative, } \\
n=144\end{array}$ & \\
\hline Age, y, mean \pm SEM & $45.7 \pm 2.7$ & $48.6 \pm 0.8$ & $43.55 \pm 2.5$ & $48.48 \pm 1.2$ & $56.6 \pm 1.7 \dagger$ \\
\hline Fem & 52.6 & 6 & 82.8 & 76.4 & 44.0 \\
\hline Hisp & 7.9 & & 55.2 & $16.7 \ddagger$ & ailable \\
\hline White, \% & 59.2 & $81.7 \S$ & 75.9 & 75.0 & Data not available \\
\hline \multicolumn{6}{|c|}{$\begin{array}{l}\text { *DPH, Connecticut Department of Public Health. } \\
\text { †Mean age for DPH case-patients differed statistically from that for seropositive nonimmunosuppressed }(p<0.001) \text { and seropositive immunosuppressed } \\
(p<0.001) \text { participants. } \\
\text { fDifference in percentage of Hispanics within the seropositive and seronegative categories of the immunosuppressed population }(p<0.0001) . \\
\S D i f f e r e n c e \text { in percentage of participants self-identifying as white within the seropositive and seronegative categories of the nonimmunosuppressed } \\
\text { population }(p<0.0001) \text {. }\end{array}$} \\
\hline
\end{tabular}

Seroconversion rates among immunosuppressed persons did not differ statistically according to sex or age. The seroconversion rate was higher among immunosuppressed Hispanics $(16 / 40,40.0 \%)$ than non-Hispanics (13/132, $9.8 \%)(\mathrm{p}<0.0001)$. Because the immunosuppression status of DPH-reported case-patients was not available, we could not further explore a role for immunosuppression in the occurrence of WNV infection among these patients. Immunosuppression carries unique risks for infectious diseases; thus, the higher rate of seroconversion among immunosuppressed participants may be a consequence of underlying medical conditions or medication regimens.

The mean age for asymptomatic seropositive adults, nonimmunosuppressed and immunosuppressed, was lower than that for DPH-reported symptomatic case-patients (Table; $p=0.0004)$. The 2 groups did not vary significantly according to sex $(p=0.30)$. Because racial data for DPH-reported case-patients was not available, no comparison by race was possible. Comparison of geocoded household locations of all study participants and DPH case-patients showed an overlapping distribution of nonimmunosuppressed and immunosuppressed asymptomatic seroconverters and DPH case-patients (online Technical Appendix Figure, https://wwwnc.cdc.gov/EID/ article/23/4/16-1669-Techapp1.pdf). Although only a surrogate for location where infection was acquired, this mapping provides no support for localized pockets of increased disease susceptibility.

We provide evidence of WNV exposure in Connecticut among 1,063 adults who differed by age, sex, race, and immunosuppression status. Among nonimmunosuppressed asymptomatic participants, age was not a significant factor with regard to WNV seroconversion. However, mean age of symptomatic case-patients was older than that of asymptomatic seropositive participants, indicating that age remains a factor in disease susceptibility (9). Age has a well-documented role in decreased immune cell function and increased susceptibility to infectious diseases, including WNV $(9,10)$; dysregulation of immune responses with elevated cytokine levels may contribute to development of severe disease. The higher WNV seroprevalence among immunosuppressed adults strongly suggests a key role for immune factors in seroconversion.

Ongoing research seeks to further define the immune system attributes that lead to increased risk for higher WNV disease severity; active areas of interest include genomic, transcriptional, and immune- and age-related variable responses $(6,8,9)$. In addition to environmental conditions that affect vector abundance, our study suggests that individual variation, such as immune status, may be a key driver for susceptibility to infection and disease severity and for differing seroconversion rates among neighbors.

\section{Acknowledgments}

We are grateful to Barbara Siconolfi, Sui Tsang, and Qiong Zhang for sample collection and helpful discussions. We thank Michael Diamond for his kind gift of recombinant WNV-specific mutant envelope protein.

This work was supported in part by awards from the National Institutes of Health (HHS N272201100019C and AI08992), the Centers for Disease Control and Prevention (U50/CCU11680601-1), the US Department of Agriculture (58- 6615-1-218, CONH00768, and CONH00773), and the Multistate Research Project (NE1043).

All procedures contributing to this work comply with the ethical standards of the relevant national and institutional committees on human experimentation and with the Helsinki Declaration of 1975 , as revised in 2008. Certain data used in this study were obtained from the Connecticut DPH, which approved this study.

Ms. Cahill is a candidate for a $\mathrm{PhD}$ degree in the epidemiology of microbial diseases at the Yale School of Public Health. She is interested in biology and viral disease susceptibility.

\section{References}

1. Centers for Disease Control and Prevention. West Nile virus, final cumulative maps \& data for 1999-2014 [cited 2016 Apr 6]. http://www.cdc.gov/westnile/statsMaps/cumMapsData.html

2. Petersen LR, Carson PJ, Biggerstaff BJ, Custer B, Borchardt SM, Busch MP. Estimated cumulative incidence of West Nile virus infection in US adults, 1999-2010. Epidemiol Infect. 2013;141:591-5. http://dx.doi.org/10.1017/S0950268812001070 
3. Suthar MS, Pulendran B. Systems analysis of West Nile virus infection. Curr Opin Virol. 2014;6:70-5. http://dx.doi.org/10.1016/ j.coviro.2014.04.010

4. Diuk-Wasser MA, Brown HE, Andreadis TG, Fish D. Modeling the spatial distribution of mosquito vectors for West Nile virus in Connecticut, USA. Vector Borne Zoonotic Dis. 2006;6:283-95. http://dx.doi.org/10.1089/vbz.2006.6.283

5. Dunne DW, Shaw A, Bockenstedt LK, Allore HG, Chen S, Malawista SE, et al. Increased TLR4 expression and downstream cytokine production in immunosuppressed adults compared to non-immunosuppressed adults. PLoS One. 2010;5:e11343. http://dx.doi.org/10.1371/journal.pone.0011343

6. Qian F, Goel G, Meng H, Wang X, You F, Devine L, et al. Systems immunology reveals markers of susceptibility to West Nile virus infection. Clin Vaccine Immunol. 2015;22:6-16. http://dx.doi.org/ 10.1128/CVI.00508-14

7. Chabierski S, Barzon L, Papa A, Niedrig M, Bramson JL, Richner JM, et al. Distinguishing West Nile virus infection using a recombinant envelope protein with mutations in the conserved fusion-loop. BMC Infect Dis. 2014;14:246. http://dx.doi.org/ 10.1186/1471-2334-14-246

8. Qian F, Thakar J, Yuan X, Nolan M, Murray KO, Lee WT, et al. Immune markers associated with host susceptibility to infection with West Nile virus. Viral Immunol. 2014;27:39-47. http://dx.doi.org/10.1089/vim.2013.0074

9. Montgomery RR. Age-related alterations in immune responses to West Nile virus infection. Clin Exp Immunol. 2017;187:26-34.

10. Shaw AC, Goldstein DR, Montgomery RR. Age-dependent dysregulation of innate immunity. Nat Rev Immunol. 2013;13: 875-87. http://dx.doi.org/10.1038/nri3547

Address for correspondence: Ruth R. Montgomery, Department of Internal Medicine, Yale University School of Medicine, 300 Cedar St/TAC S413, New Haven, CT 06520-8031, USA; email: ruth.montgomery@yale.edu

\section{mcr-1 in Enterobacteriaceae from Companion Animals, Beijing, China, 2012-2016}

\section{Lei Lei, ${ }^{1}$ Yang Wang, ${ }^{1}$ Stefan Schwarz, Timothy R. Walsh, Yanran Ou, Yifan Wu, Mei Li, Zhangqi Shen}

Author affiliations: China Agricultural University, Beijing, China (L. Lei, Y. Wang, Y. Ou, Y. Wu, M. Li, Z. Shen); Freie Universität Berlin, Berlin, Germany (S. Schwarz); Cardiff University, Cardiff, Wales, UK (T.R. Walsh, M. Li); lowa State University, Ames, lowa, USA (Z. Shen)

DOI: http://dx.doi.org/10.3201/eid2304.161732

${ }^{1}$ These authors contributed equally to this article.
To investigate the prevalence of the recently emerging colistin resistance gene mcr-1 in Enterobacteriaceae among companion animals, we examined 566 isolates collected from cats and dogs in Beijing, China, during 2012-2016. Of these isolates, $49(8.7 \%)$ were $m c r-1$-positive.

$\mathrm{M}$ ultidrug-resistant and extensively drug-resistant gramnegative bacteria are a major threat to public health worldwide $(1,2)$. The recent rapid dissemination of carbapenem-resistant Enterobacteriaceae has worsened this situation and further narrowed treatment options for infections caused by these bacteria (3). Colistin is a last-resort drug for treating carbapenem-resistant Enterobacteriaceae infections (4). In 2016, we identified the mobile colistin resistance gene $m c r-1$ (1). Soon after its description, $m c r-1$ was observed in Enterobacteriaceae from humans and food-producing animals in $>30$ countries on 5 continents (5).

A 2016 article reported that a 50-year-old man who worked in a pet store tested positive for $\mathrm{mcr}$ - 1 -harboring E. coli (6). Investigation identified 6 multidrug-resistant $m c r-1$-producing $E$. coli isolates in samples from 4 dogs and 2 cats in the pet store, indicating that the pathogens can be transmitted between humans and companion animals. So far, the prevalence of $\mathrm{mcr}$-1-containing Enterobacteriaceae in companion animals is largely unknown. In our study, we focused on estimating the prevalence of $\mathrm{mcr}-1$ in Enterobacteriaceae of companion animal origin in Beijing, China, during 2012-2016, and investigated the presence of the $m c r-1$ gene in pet foods purchased there.

In Beijing, the total number of registered dogs and cats is $\approx 1.2$ million. We collected samples from both healthy and sick dogs and cats in Veterinary Teaching Hospital of China Agricultural University.

A total of 566 nonduplicate Enterobacteriaceae strains were isolated from 1,439 nasal and rectal swab samples collected from 1,254 dogs and 185 cats during 2012-2016. We also isolated 25 Enterobacteriaceae from 32 nasal swab samples from the pet owners. Because the food chain is among the main routes for humans and companion animals to acquire foodborne pathogens, we collected a small sample of pet foods ( $\operatorname{dog}$ food, $\mathrm{n}=30$; cat food, $\mathrm{n}=5$ ) containing chicken as the main ingredient in Beijing during June-August 2016.

The species of all Enterobacteriaceae were determined by $16 \mathrm{~S}$ rDNA sequencing and matrix-assisted laser desorption/ionization time-of-flight mass spectrometry of specimens cultured on brain-heart infusion agar plates containing $2 \mu \mathrm{g} / \mathrm{mL}$ colistin. A total of 79/566 (14.0\%) of the Enterobacteriaceae isolates from companion animals were resistant to colistin: 56 E. coli, 16 Klebsiella pneumoniae, 5 Enterobacter cloacae, 1 Enterobacter aerogenes, and 1 Shigella spp. PCR amplification of $m c r-1$ indicated that 8.7\% (49/566) of Enterobacteriaceae and 62.0\% (49/79) 